\title{
Dipeptidyl Peptidase-4 Inhibitors and COVID-19-Related Deaths among Patients with Type 2 Diabetes Mellitus: A Meta-Analysis of Observational Studies
}

\author{
Dimitrios Patoulias ${ }^{1}$, Michael Doumas ${ }^{1,2}$ \\ ${ }^{1}$ Second Propedeutic Department of Internal Medicine, General Hospital "Hippokration”, Aristotle University of Thessaloniki, \\ Thessaloniki, Greece; ${ }^{2}$ Veterans Affairs Medical Center, George Washington University, Washington, DC, USA
}

The coronavirus disease 2019 (COVID-19) pandemic remains an unbeaten enemy. Unfortunately, no targeted treatment option is available. Patients with type 2 diabetes mellitus (T2DM) have increased odds for severe or fatal disease, as demonstrated in recent observational studies. There is an ongoing discussion regarding the impact of different antidiabetic drug classes on outcomes of interest among affected subjects. Dipeptidyl peptidase-4 (DPP-4) inhibitors have been placed at the epicenter, since the DPP-4 enzyme seems to be implicated in the disease pathogenesis. Herein we present an updated meta-analysis of observational studies addressing the risk of COVID-19 death among patients with T2DM on prior DPP-4 inhibitor treatment. We pooled data from 10 observational studies, showing that DPP-4 inhibitors produce a non-significant decrease in the risk for COVID-19-related death. However, when administered in the inpatient setting, DPP-4 inhibitors decrease the risk for COVID-19-related death by 50\%. Ongoing randomized controlled trials will shed further light.

Keywords: Dipeptidyl-peptidase IV inhibitors; Diabetes mellitus, type 2; COVID-19; Mortality

\section{INTRODUCTION}

The coronavirus disease 2019 (COVID-19) pandemic has accounted for more than 108 million documented cases and 2 million deaths worldwide as of February 2021. Patients with diabetes mellitus have a significantly higher risk for severe disease and COVID-19-related death than the general population, representing a very sensitive population group $[1,2]$. A vivid and ongoing discussion is continuing regarding the place of antidiabetic drug classes in the therapeutic management of COVID19-infected patients with type 2 diabetes mellitus (T2DM) and whether antidiabetic medications predispose patients to infec-

Received: 26 March 2021, Revised: 3 June 2021, Accepted: 8 June 2021

Corresponding author: Dimitrios Patoulias

Second Propedeutic Department of Internal Medicine, General Hospital

"Hippokration", Konstantinoupoleos 49, 54642, Thessaloniki, Greece

Tel: +30-6946900777, Fax: +30-2310225083, E-mail: dipatoulias@gmail.com tions [3].

Dipeptidyl peptidase-4 (DPP-4) inhibitors have attracted scientific interest due to the potential involvement of the DPP-4 enzyme (also known as the T-cell antigen CD26) in the pathophysiology of the infection, along with the anti-inflammatory properties of this drug class [4]. A previously published metaanalysis of six observational studies by Kow and Hasan [5] addressing the composite outcome of severe or fatal illness demonstrated that pre-admission use of DPP-4 inhibitors did not have a significant impact on prespecified outcomes. More recently, Bonora et al. [6] pooled data from seven observational studies published in the Journal of Endocrinological Investiga-

Copyright $\odot 2021$ Korean Endocrine Society

This is an Open Access article distributed under the terms of the Creative Commons Attribution Non-Commercial License (https://creativecommons.org/ licenses/by-nc/4.0/) which permits unrestricted non-commercial use, distribution, and reproduction in any medium, provided the original work is properly cited. 
tion, demonstrating that the prior use of DPP-4 inhibitors was not associated with an increased risk of COVID-19-related death, having a rather neutral effect. The aim of the present study was to update the previously published meta-analyses.

\section{METHODS}

We sought to determine whether DPP-4 inhibitors influence the risk for COVID-19-related death, which corresponds to the worst outcome of the disease course. We searched two major databases (PubMed and Cochrane Library) for published randomized controlled trials (RCTs) or observational studies enrolling adult patients with T2DM with prior use of DPP-4 inhibitors versus other antidiabetic drug classes, with confirmed $\mathrm{CO}$ VID-19 infection, by applying the following search strategy: (sitagliptin OR vildagliptin OR alogliptin OR saxagliptin OR linagliptin OR anagliptin OR tenegliptin OR trelagliptin OR gemigliptin OR DPP-4 inhibitor) AND (COVID-19 OR SARSCoV-2 OR covid19). We excluded studies of the pediatric population and studies enrolling patients with type 1 diabetes mellitus and patients with suspected COVID-19 infection without laboratory confirmation. We did not apply any limitation according to publication language or study sample size. We handsearched the retrieved bibliographies to identify potentially eligible studies that were missed with the applied search strategy.

Two independent reviewers (D.P. and M.D.) extracted the data from the eligible reports using a pilot-tested data extraction form. We set a "hard" safety outcome of COVID-19-related death (regardless of occurrence in the outpatient or inpatient setting).

As we only assessed a dichotomous variable, the difference was calculated using risk ratios (RRs) with 95\% confidence intervals (CIs), after implementation of the Mantel-Haenszel random effects formula. Statistical heterogeneity among studies was assessed using the $I^{2}$ statistic [7]. All analyses were performed at the 0.05 significance level using RevMan version 5.3 software (Nordic Cochrane Centre, Copenhagen, Denmark).

\section{RESULTS}

Our search yielded a total of 77 reports. After deduplication and screening, we retrieved 17 reports for potential inclusion in our meta-analysis. We finally included in our quantitative synthesis 10 observational studies, published until May 5, 2021, while no relevant RCTs were identified [8-17]. The detailed selection of studies is provided in detail in the corresponding flow diagram (Supplemental Fig. S1). Only two studies evaluated the effect of DPP-4 inhibitors in the inpatient setting $[8,11]$, while the others assessed COVID-19 surrogate outcomes in diabetes patients who had previously used DPP-4 inhibitors in the outpatient setting [9,10,12-17].

We therefore pooled data from 10 observational studies. The

\begin{tabular}{|c|c|c|c|c|c|}
\hline \multirow[b]{2}{*}{ Study or Subgroup } & \multicolumn{2}{|c|}{ DPP.4 inhibitor } & \multicolumn{2}{|c|}{ Control } & \multirow[b]{2}{*}{ Weight M-r } \\
\hline & Events & Total & Events & Total & \\
\hline \multicolumn{6}{|c|}{ 1.1.1 Outpatient setting } \\
\hline Chen 2020 & 5 & 20 & 14 & 100 & $8.3 \%$ \\
\hline Fadini 2020 & 1 & 9 & 10 & 76 & $3.0 \%$ \\
\hline Israelsen 2021 & 30 & 284 & 21 & 644 & $12.2 \%$ \\
\hline Mirani 2020 & 1 & 11 & 37 & 79 & $3.1 \%$ \\
\hline Noh 2021 & 47 & 453 & 22 & 133 & $13.0 \%$ \\
\hline Pérez-Belmonte 2020 & 75 & 180 & 440 & 1409 & $15.8 \%$ \\
\hline Silverii 2020 & 5 & 13 & 54 & 146 & $10.1 \%$ \\
\hline Wargny 2021 & 113 & 615 & 464 & 2179 & $15.9 \%$ \\
\hline Subtotal $(95 \% \mathrm{Cl})$ & & 1585 & & 4766 & $81.4 \%$ \\
\hline Total events & 277 & & 1062 & & \\
\hline \multicolumn{6}{|c|}{$\begin{array}{l}\text { Heterogeneity. } \text { Tau }^{2}=0.17 ; \mathrm{Chi}^{2}=36.37, \mathrm{df}=7(P<0.00001) ;\left.\right|^{2}=81 \% \\
\text { Test for overall effect: } Z=0.67(P=0.50)\end{array}$} \\
\hline \multicolumn{6}{|l|}{ 1.1.2 Inpatient setting } \\
\hline Solerte 2020 & 31 & 169 & 63 & 169 & $14.1 \%$ \\
\hline Zhou 2021 & 2 & 111 & 11 & 333 & $4.4 \%$ \\
\hline Subtotal $(95 \% \mathrm{Cl})$ & & 280 & & 502 & $18.6 \%$ \\
\hline Total events & 33 & & 74 & & \\
\hline \multicolumn{6}{|c|}{$\begin{array}{l}\text { Heterogeneity: } \mathrm{Tau}^{2}=0.00 ; \mathrm{Chi}^{2}=0.02, \mathrm{df}=1(P=0.89) ;\left.\right|^{2}=0 \% \\
\text { Test for overall effect: } Z=3.80(P=0.0001)\end{array}$} \\
\hline Total $(95 \% \mathrm{Cl})$ & & 1865 & & 5268 & $100.0 \%$ \\
\hline Total events & 310 & & 1136 & & \\
\hline \multicolumn{6}{|c|}{$\begin{array}{l}\text { Heterogeneity: } \operatorname{Tau}^{2}=0.21 ; \mathrm{Chi}^{2}=52.99, \mathrm{df}=9(P<0.00001) ;\left.\right|^{2}=83 \% \\
\text { Test for overall effect: } Z=0.14(P=0.89)\end{array}$} \\
\hline
\end{tabular}

Risk Ratio Risk Ratio M-H, Random, $95 \% \mathrm{Cl}$

$1.79[0.73,4.40]$

$0.84[0.12,5.85]$

$3.24[1.89,5.56]$

$0.19[0.03,1.28]$

$0.63[0.39,1.00]$

$1.33[1.10,1.61]$

$1.04[0.51,2.14]$

$0.86[0.72,1.04]$

$1.14[0.78,1.66]$

Cl
40]
85]
56]
$28] ~$
00]
$61]$
$14]$
04]
$66]$

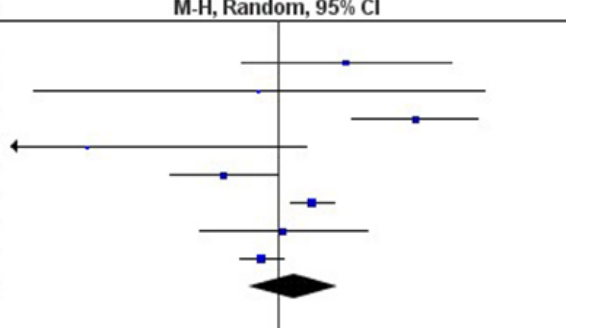

$0.49[0.34,0.71]$ $0.55[0.12,2.42]$ $0.50[0.34,0.71]$

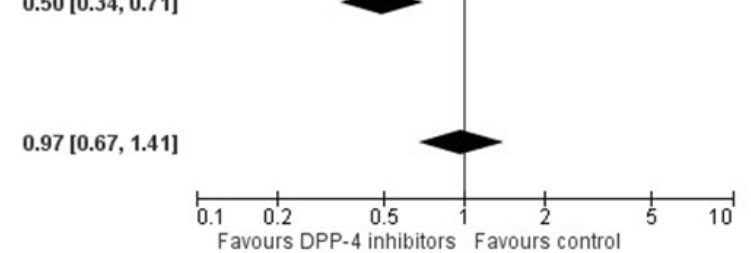

Fig. 1. Effect of dipeptidyl peptidase-4 (DPP-4) inhibitors compared to control on the risk of coronavirus disease 2019 (COVID-19)-related death. M-H, Mantel-Haenszel; CI, confidence interval. 
main baseline characteristics across the included studies are summarized in Supplemental Table S1. The use of DPP-4 inhibitors compared to other antidiabetic drug classes produced a nonsignificant decrease of 3\% in the risk for COVID-19 related death ( $95 \% \mathrm{CI}, 0.67$ to $\left.1.41 ; I^{2}=83 \% ; P=0.89\right)$, as shown in Fig. 1. Of note, subgroup analyses according to the setting revealed that, when DPP- 4 inhibitors were initiated in the inpatient setting, the result became significant (RR, $0.50 ; 95 \% \mathrm{CI}, 0.34$ to $0.71 ; I^{2}=0 \% ; P=0.0001$ ), while outpatient use of this antidiabetic drug class was not associated with a significant effect on the risk for COVID-19 death (RR, 1.14; 95\% CI, 0.78 to 1.66; $I^{2}=81 \% ; P=0.50$ ), as clearly depicted in Fig. 1 . Testing for subgroup differences revealed a significant difference between inpatient and outpatient use of DPP-4 inhibitors $(P=0.002)$.

\section{Strengths and limitations}

Herein we present the most recent, updated, relevant meta-analysis addressing the potential impact of DPP-4 inhibitors on COVID-19-related death. We performed a sub-analysis according to the setting of DPP-4 administration (inpatient vs. outpatient). Major limitations of the present meta-analysis include the small number of included trials, the absence of RCTs, the small number of enrolled subjects across eligible studies, and the high observed heterogeneity for the assessed safety outcome.

\section{DISCUSSION}

Human DPP-4 has been identified as a transmembrane glycoprotein acting as a functional receptor for the spike protein of coronaviruses, including Middle East respiratory syndrome coronavirus (MERS-CoV) and severe acute respiratory syndrome coronavirus 2 (SARS-CoV-2), and playing a crucial role in immune regulation, as it is implicated in the activation of Tcells and upregulation of the CD86 and nuclear factor- $\mathrm{\kappa B}$ pathways [18]. Therefore, it may be deduced that DPP-4 might be involved in the cytokine storm and the hyperinflammation observed among patients infected with SARS-CoV-2. It has been previously shown that DPP-4 inhibitors exert significant systemic anti-inflammatory effects, finding with direct implications for COVID-19, by decreasing the concentration of major inflammatory cytokines, such as interleukin-6 (IL-6) [19]. In addition, DPP-4 inhibition has been shown to significantly suppress pulmonary hyperinflammation in acute lung injury, as observed in a relevant experimental model, in which sitagliptin suppressed the lipopolysaccharide-induced lung injury in mice by decreasing the release of cytokines, such as tumor necrosis factor-alpha (TNF- $\alpha$ ) and IL-6 by human lung microvascular endothelial cells [20]. To summarize, it seems that there is a strong pathophysiological basis supporting the hypothesis that DPP-4 inhibition can decrease major SARS-CoV-2 complications, such as acute respiratory distress syndrome, which represents the main cause of death in COVID-19 patients. Human mechanistic studies are required to confirm this hypothesis.

Regarding the observed significant effect of the inpatient usage of DPP-4 inhibitors on the risk for COVID-19-related death, the results are based upon only two studies, primarily driven by the results of the observational study conducted by Solerte et al. [8]. More specifically, Solerte et al. [8] demonstrated that sitagliptin treatment compared to standard of care resulted in a significant reduction in $\mathrm{C}$-reactive protein $(P=0.001)$ and procalcitonin $(P=0.01)$ levels at follow-up (30 days post-admission), while no significant difference in levels of other inflammatory markers, such as ferritin and IL-6, was documented. The researchers suggested an acute anti-inflammatory and immunomodulatory effect of sitagliptin, potentially preventing multi-organ failure and associated complications. However, in another study conducted in the inpatient setting, Zhou et al. [11] showed that inpatient usage of DPP-4 inhibitors compared to control did not have a significant effect on neutrophil count, IL-6 and TNF- $\alpha$, raising doubts regarding whether DPP-4 inhibitors have a true anti-inflammatory effect in COVID-19 patients in the acute setting. Despite these observations, it is important to keep in mind that our results are based only on two studies. Future relevant studies assessing the efficacy of inpatient administration of DPP-4 inhibitors will provide mechanistic insights that could potentially explain these results.

Collectively, despite the fact that current evidence is based upon observational studies, prior use of DPP-4 inhibitors has a neutral effect on COVID-19-related deaths among patients with T2DM, while inpatient administration might contribute to a decrease in the corresponding risk. Ongoing phase 3 RCTs will shed further light on the impact of this antidiabetic drug class on surrogate safety endpoints among patients hospitalized with COVID-19 (ClinicalTrials.gov Identifier: NCT04365517, NCT04371978). According to current knowledge, DPP-4 inhibitors should not be discontinued in infected patients.

\section{CONFLICTS OF INTEREST}

No potential conflict of interest relevant to this article was reported. 


\section{AUTHOR CONTRIBUTIONS}

Conception or design: D.P., M.D. Acquisition, analysis, or interpretation of data: D.P., M.D. Drafting the work or revising: D.P., M.D. Final approval of the manuscript: D.P., M.D.

\section{ORCID}

Dimitrios Patoulias https://orcid.org/0000-0002-6899-684X

\section{REFERENCES}

1. Aggarwal G, Lippi G, Lavie CJ, Henry BM, Sanchis-Gomar F. Diabetes mellitus association with coronavirus disease 2019 (COVID-19) severity and mortality: a pooled analysis. J Diabetes 2020;12:851-5.

2. Varikasuvu SR, Dutt N, Thangappazham B, Varshney S. Diabetes and COVID-19: a pooled analysis related to disease severity and mortality. Prim Care Diabetes 2021;15:24-7.

3. Lim S, Bae JH, Kwon HS, Nauck MA. COVID-19 and diabetes mellitus: from pathophysiology to clinical management. Nat Rev Endocrinol 2021;17:11-30.

4. Scheen AJ. DPP-4 inhibition and COVID-19: from initial concerns to recent expectations. Diabetes Metab 2021;47: 101213

5. Kow CS, Hasan SS. A meta-analysis on the preadmission use of DPP-4 inhibitors and risk of a fatal or severe course of illness in patients with COVID-19. Therapie 2021;76:361-4.

6. Bonora BM, Avogaro A, Fadini GP. Disentangling conflicting evidence on DPP-4 inhibitors and outcomes of COVID-19: narrative review and meta-analysis. J Endocrinol Invest 2021;44:1379-86.

7. Higgins JPT, Green S. Cochrane handbook for systematic reviews of interventions, version 5.1.0. Chichester: John Wiley \& Sons Ltd.; 2011. Chapter 9, Analyzing data and undertaking meta-analyses. Available from: https:/handbook-5-1.cochrane.org.

8. Solerte SB, D'Addio F, Trevisan R, Lovati E, Rossi A, Pastore I, et al. Sitagliptin treatment at the time of hospitalization was associated with reduced mortality in patients with type 2 diabetes and COVID-19: a multicenter, case-control, retrospective, observational study. Diabetes Care 2020;43: 2999-3006.

9. Noh Y, Oh IS, Jeong HE, Filion KB, Yu OH, Shin JY. Association between DPP-4 inhibitors and COVID-19-related outcomes among patients with type 2 diabetes. Diabetes
Care 2021;44:e64-6.

10. Israelsen SB, Pottegard A, Sandholdt H, Madsbad S, Thomsen RW, Benfield T. Comparable COVID-19 outcomes with current use of GLP-1 receptor agonists, DPP-4 inhibitors or SGLT-2 inhibitors among patients with diabetes who tested positive for SARS-CoV-2. Diabetes Obes Metab 2021;23: 1397-401.

11. Zhou JH, Wu B, Wang WX, Lei F, Cheng X, Qin JJ, et al. No significant association between dipeptidyl peptidase-4 inhibitors and adverse outcomes of COVID-19. World J Clin Cases 2020;8:5576-88.

12. Perez-Belmonte LM, Torres-Pena JD, Lopez-Carmona MD, Ayala-Gutierrez MM, Fuentes-Jimenez F, Huerta LJ, et al. Mortality and other adverse outcomes in patients with type 2 diabetes mellitus admitted for COVID-19 in association with glucose-lowering drugs: a nationwide cohort study. BMC Med 2020;18:359.

13. Mirani M, Favacchio G, Carrone F, Betella N, Biamonte E, Morenghi E, et al. Impact of comorbidities and glycemia at admission and dipeptidyl peptidase 4 inhibitors in patients with type 2 diabetes with COVID-19: a case series from an academic hospital in Lombardy, Italy. Diabetes Care 2020; 43:3042-9.

14. Fadini GP, Morieri ML, Longato E, Bonora BM, Pinelli S, Selmin E, et al. Exposure to dipeptidyl-peptidase-4 inhibitors and COVID-19 among people with type 2 diabetes: a case-control study. Diabetes Obes Metab 2020;22:1946-50.

15. Wargny M, Potier L, Gourdy P, Pichelin M, Amadou C, Benhamou PY, et al. Predictors of hospital discharge and mortality in patients with diabetes and COVID-19: updated results from the nationwide CORONADO study. Diabetologia 2021;64:778-94.

16. Chen Y, Yang D, Cheng B, Chen J, Peng A, Yang C, et al. Clinical characteristics and outcomes of patients with diabetes and COVID-19 in association with glucose-lowering medication. Diabetes Care 2020;43:1399-407.

17. Silverii GA, Monami M, Cernigliaro A, Vigneri E, Guarnotta $\mathrm{V}$, Scondotto $\mathrm{S}$, et al. Are diabetes and its medications risk factors for the development of COVID-19?: data from a population-based study in Sicily. Nutr Metab Cardiovasc Dis 2021;31:396-8.

18. Iacobellis G. COVID-19 and diabetes: can DPP4 inhibition play a role? Diabetes Res Clin Pract 2020;162:108125.

19. Rizzo MR, Barbieri M, Marfella R, Paolisso G. Reduction of oxidative stress and inflammation by blunting daily acute glucose fluctuations in patients with type 2 diabetes: role of 
dipeptidyl peptidase-IV inhibition. Diabetes Care 2012;35: 2076-82.

20. Kawasaki T, Chen W, Htwe YM, Tatsumi K, Dudek SM.
DPP4 inhibition by sitagliptin attenuates LPS-induced lung injury in mice. Am J Physiol Lung Cell Mol Physiol 2018; 315:L834-45. 